\title{
MedienPädagogik
}

Zeitschrift für Theorie und Praxis der Medienbildung

Themenheft Nr. 37: Medienpädagogik als Schlüsseldisziplin in einer mediatisierten Welt. Perspektiven aus Theorie, Empirie und Praxis Herausgegeben von Henrike Friedrichs-Liesenkötter, Lara Gerhardts, Anna-Maria Kamin und Sonja Kröger

\section{Kompetenzerwartungen an Lehrpersonen und Professionalisierung angesichts von Mediatisierung und Digitalisierung}

Gerhard Tulodziecki und Silke Grafe

\section{Zusammenfassung}

Mit der Mediatisierung und Digitalisierung und dem damit verbundenen gesellschaftlichen Wandel entstehen neue Kompetenzanforderungen an Aufwachsende und Lehrpersonen. Dafür bedarf es zunächst einer zeitgemässen Sicht auf Zielvorstellungen, mediale Nutzungsformen und Handlungsbereiche, medienbezogene Inhalte und geeignete Vorgehensweisen sowie Aufgabenfelder für die Medienbildung. Auf dieser Grundlage werden in dem Beitrag vor dem Hintergrund der deutschsprachigen Diskussion um medienpädagogische Kompetenz Aufgabenfelder und Kompetenzerwartungen für die Lehrerinnen- und Lehrerbildung formuliert. Dabei lassen sich einzelne Gemeinsamkeiten und Unterschiede zur Diskussion im internationalen Raum feststellen. Insgesamt sollte der Kompetenzerwerb von Lehrpersonen an Merkmalen professionellen Handelns orientiert sein und im Kontext von Professionalisierung gesehen werden. Dies macht für Lehrpersonen u.a. die Verfügung über geeignete wissenschaftliche Grundlagen für medienpädagogisch relevantes Handeln, die Fähigkeit zum In-BeziehungSetzen von wissenschaftlich fundiertem Wissen und praktischen Situationen, die Entwicklung einer forschenden Grundeinstellung und einer berufsethischen Haltung notwendig. Der Aufbau entsprechender Dispositionen bedarf eines län- 
gerfristigen Prozesses, der im Sinne von Professionalisierung als Entwicklung von der Novizin bzw. vom Novizen zur Expertin bzw. zum Experten beschrieben werden kann.

\title{
Competence Expectations for Teachers and Professionalization in the Light of Mediatization and Digitalization
}

\begin{abstract}
Due to processes of mediatization and digitalization and the resulting societal transformation, new demands emerge with regards to the competencies of adolescents and educators. This context necessitates a contemporary perspective on objectives, fields of media use and media actions, media-related contents and suitable procedures as well as tasks for media education. On this basis and against the background of the discourse about «medienpädagogische Kompetenz> [media pedagogical competence] in German-speaking countries, tasks and competence expectations for teacher education can be framed. In this context, there are certain similarities and differences to the respective international discourse. Overall, teachers' competency acquisition should be oriented towards aspects of professional acting and be understood in the context of professionalization. Among other things, this requires educators to have the appropriate scientific basics for actions of media pedagogical relevance at their disposal, to be able to correlate scientifically grounded knowledge with practical situations, and to develop an orientation towards research and a professional ethical attitude. Building such dispositions requires a long-term process. In the sense of professionalization, this process can be described as the development from novice to expert.
\end{abstract}




\section{Mediatisierung und Digitalisierung als Herausforderung für die Schule}

Die digitale Technik führt im Zusammenhang mit Mediatisierungsprozessen zu einem fundamentalen Wandel des gesamten Mediensystems. Der Wandel zeigt sich u.a. im Aufkommen neuer Medien, z.B. des Smartphones mit all seinen Möglichkeiten, in der Veränderung vorhandener Medien, z.B. von Fernsehen und Radio, sowie in dem Übergang von relativ unabhängigen Einzelmedien zu einer umfassenden digitalen Infrastruktur, wobei alle Entwicklungen durch ökonomische Interessen vorangetrieben werden (Krotz 2016, 25-31). Im Vergleich zu früheren Mediatisierungsprozessen, z.B. aufgrund der Erfindung und Verbreitung des Buchdrucks, des Films oder des Fernsehens, hat die gegenwärtige Form der Mediatisierung ihren Ursprung darin, dass die in einer medialen Botschaft enthaltene Information in maschinell verarbeitbare Daten umgewandelt wird. Beispielsweise lässt sich jeder Buchstabe bzw. schriftliche Text in Form von Binärziffern (binary digits: $\mathrm{O}$ und 1) ausdrücken. Gleiches gilt für Bilder und Töne. Die Binärziffern sind physikalisch darstellbar, z.B. als geringe oder hohe elektrische Spannung, und können so maschinell verarbeitet werden. Die algorithmisch bzw. maschinell verarbeiteten Daten lassen sich dann wieder in Form von schriftlichen Texten, Bildern oder Tönen präsentieren, so dass Menschen ihnen eine Bedeutung zumessen und sie als Information bzw. Botschaft interpretieren können. Durch entsprechende maschinelle Prozesse lässt sich nicht nur Vorgegebenes in anderer Form verbinden und präsentieren, es können auch neue schriftliche Texte, Bilder und Töne oder allgemeiner: zeichenfähige bzw. interpretationsfähige Muster - entstehen (Herzig 2012, 1:102-8 u. 173-75).

Wandlungsprozesse, die durch die Nutzung digitaler Techniken bedingt sind, werden üblicherweise und allgemein mit dem Begriff der Digitalisierung bezeichnet. Damit hat der Begriff, der ursprünglich nur die Umwandlung analoger Grössen in binäre Werte oder digitale Repräsentationen meinte, eine deutliche Erweiterung im Begriffsverständnis erfahren (Knaus 2016, 101-2). Seine Bedeutung ist auch damit verbunden, dass die digitale Technik Vernetzung, Sensorisierung, Datafizierung und Algorithmisierung ermöglicht (Gapski 2016, 22). Im Kontext solcher Möglichkeiten hat die Digitalisierung den eingangs angesprochenen Mediatisierungsschub 
ausgelöst. Dieser ist auf die oben genannten früheren Formen der Mediatisierung gestossen und hat zusammen mit der Digitalisierung mannigfaltige gesellschaftlichen Folgen, die weit über technische Fragen hinausgehen und den gesellschaftlichen Wandel betreffen (Krotz und Hepp 2012, 12).

Mit der Entwicklung der Medienlandschaft und ihrer digitalen Infrastruktur haben sich die Kompetenzanforderungen an Kinder, Jugendliche und Erwachsene deutlich verändert: Das Verstehen der 〈Sprache〉 der Medien und die eigene Ausdrucksfähigkeit in dieser 〈Zeichensprache〉 gelten mittlerweile als wichtige Kulturtechniken. Darüber hinaus wird es für die Teilhabe am beruflichen, kulturellen und gesellschaftlichen Leben immer wichtiger, die hinter den medialen Möglichkeiten liegende digitale Infrastruktur sowie die Einflüsse von Medien auf Individuum und Gesellschaft und die Bedingungen ihrer Produktion und Verbreitung zu durchschauen und kritisch einzuschätzen. In diesem Zusammenhang geht es in der Schule darum sicherzustellen, dass alle Schülerinnen und Schüler eine zeitgemässe Medienbildung erhalten. Dies setzt eine entsprechende Kompetenz und Professionalisierung von Lehrpersonen voraus.

Vor diesem Hintergrund stellt sich zunächst die Frage, wie eine zeitgemässe Medienbildung charakterisiert werden kann und welche Aufgabenfelder sich dabei abzeichnen. Danach ist zu fragen, welche Kompetenzerwartungen an Lehrpersonen zu richten sind, ehe der Kompetenzerwerb unter der Perspektive von Professionalisierung zu diskutieren ist. Wir greifen damit einzelne Fragestellungen auf, die auch Dorothee Meister immer wieder beschäftigt haben (Meister und Sander 1999; Knaus, Meister, und Tulodziecki 2018).

\section{Dimensionen und Aufgabenfelder der Medienbildung als Bezugspunkte}

Eine zeitgemässe Medienbildung lässt sich durch ihre Zieldimension, ihre Dimension medialer Nutzungsformen und Handlungsbereiche, ihre Inhaltsdimension und ihre Vorgehensdimension kennzeichnen (Tulodziecki, Herzig, und Grafe 2019, 198-207):

Zieldimension: Hinsichtlich der Zieldimension gehen wir davon aus, dass auch in einer von Digitalisierung und Mediatisierung gekennzeichneten 
Welt das gesellschaftlich handlungsfähige Subjekt eine zentrale Leitidee für Erziehung und Bildung darstellt - verbunden mit den Zielperspektiven eines sachgerechten, eines selbstbestimmten, eines kreativen und eines sozial verantwortlichen Handelns. In der Auslegung dieser Zielperspektiven lassen sich als Zielbereiche nennen: Handhabungsfertigkeiten, Kommunikationsfähigkeit, Kenntnisse und Verstehen in wichtigen medienbezogenen Inhaltsbereichen, Recherche- und Strukturierungsfähigkeiten, Fähigkeit zur Analyse und Bewertung, Problemlösefähigkeit, Entscheidungsfähigkeit, Gestaltungsfähigkeit, Urteilsfähigkeit und Handlungsfähigkeit sowie Handlungsbereitschaft.

Dimension medialer Nutzungsformen und Handlungsbereiche: Im Hinblick auf eine bildungsbezogene Auseinandersetzung mit der Mediennutzung und dem Medienhandeln lassen sich zunächst verschiedene Formen der Mediennutzung nennen: die rezeptive Nutzung von Medien (z.B. bei Fernsehen oder Radio), die interaktiv-eingreifende Nutzung (z.B. beim Computerspiel), die produktive Nutzung (z.B. bei der Erstellung eines eigenen Videos), die interaktiv-austauschbezogene Nutzung (z.B. bei InstantMessaging-Diensten oder Social Networks), die interaktiv-partizipative Nutzung (z.B. bei Wikis und Blogs) und die interaktiv-steuerungsorientierte Nutzung (z.B. bei Lifelogging oder Smart Home). Die verschiedenen Nutzungsformen können im Kontext verschiedener Verwendungszwecke bzw. Handlungsbereiche zur Geltung kommen. Als solche lassen sich nennen: Information und Lernen, Analyse und Simulation, Unterhaltung und Spiel, Dienstleistung und Steuerung sowie Kontrolle.

Inhaltsdimension: Hier sind zum einen Inhaltsbereiche zu beachten, die sich in der medienpädagogischen Diskussion herausgebildet haben, z.B. Medienlandschaft, 〈Sprache〉 der Medien, Medieneinflüsse oder Produktionsbedingungen. Zum anderen muss die Medienbildung angesichts der Digitalisierung informatische Grundlagen berücksichtigen (vgl. z.B. Döbeli Honneger 2016, 63-82). Vor diesem Hintergrund gehen wir für die Medienbildung von folgenden Inhaltsbereichen aus: (a) Medienlandschaft und ihre digitale Infrastruktur, wobei mit Bezug auf die Medienlandschaft die Vielfalt der Medienarten, die Medienkonvergenz, das weite Spektrum der inhaltlichen Angebote und die verschiedenen Zugangsformen zur Sprache kommen sollten und mit Bezug auf die Infrastruktur die Überführung von 
Information in Daten, die Datenerfassung durch Eingabe oder Sensoren, die Modellierung und die algorithmische Datenverarbeitung und Datenanalyse sowie die Automatisierung von Prozessen und die Vernetzung von Informatiksystemen. (b) Gestaltungsmerkmale und Erzeugung medialer Botschaften, wobei ausgehend von einer Thematisierung des Zusammenhangs von Zeichen und Bedeutung zum einen mediale Gestaltungsmerkmale in ihrem Verhältnis zum Inhalt zu behandeln sind und zum anderen Formen der Erzeugung medialer Botschaften - von der Auftragung von Substanzen auf einen materialen Träger (beim Malen) bis zur algorithmischen Verund Bearbeitung von Daten und automatischen Generierung zeichenfähiger Muster. (c) Einflüsse von Medien auf Individuum und Gesellschaft, wobei es um medienbeeinflusste Vorstellungen und Einstellungen, Emotionen und Verhaltensmuster, Wertorientierungen mit ihren Folgen für soziale Zusammenhänge sowie um Einflüsse auf Kultur und Gesellschaft insgesamt geht. (d) Bedingungen der Medienproduktion und Medienverbreitung, wobei technische, rechtliche, ökonomische, personale, institutionelle, politische und weitere gesellschaftlich-kulturelle Bedingungen in den Blick genommen werden sollten.

Vorgehensdimension: Die Fülle möglicher Inhalte legt für die Medienbildung eine Verbindung von exemplarischem und orientierendem Vorgehen nahe. Hinsichtlich dafür geeigneter Vorgehensweisen ist es wichtig, von der Lebenssituation und der Bedürfnislage der Heranwachsenden sowie vom jeweils gegebenen Wissens-, Erfahrungs- und Entwicklungsstand auszugehen und in kommunikativer Weise eine Weiterentwicklung anzustreben. Dies sollte im Rahmen der Auseinandersetzung mit komplexen Aufgaben - einschliesslich der jeweils notwendigen Wissensaneignung - geschehen. Als geeignete Aufgabenformen können dabei Erkundungsaufgaben, Probleme, Entscheidungsfälle, Gestaltungs- oder Beurteilungsaufgaben dienen. In diesem Sinne bietet sich für die Medienbildung ein erkundungs-, problem-, entscheidungs-, gestaltungs- und beurteilungsorientiertes Vorgehen an (Tulodziecki, Herzig, und Grafe 2019, 206-7).

Versucht man auf der Grundlage dieser Dimensionen Aufgabenfelder für die Medienbildung in der Schule zu beschreiben, lassen sich verschiedene nutzungs- und inhaltsbezogene Aufgabenfelder nennen. Als nutzungsbezogene Aufgabenfelder können gelten: der kritische Umgang und die 
reflektierte Nutzung von medialen Möglichkeiten (a) für Information und Lernen, (b) für Analyse und Simulation, (c) für Unterhaltung und Spiel, (d) Austausch und Kooperation, (e) für die Gestaltung und Präsentation eigener Medienbeiträge, (f) für Dienstleistung und Steuerung. Als inhaltsbezogene Aufgabenfelder lassen sich nennen: (1) Verstehen und Bewerten der Medienlandschaft und ihrer digitalen Infrastruktur, (2) Analysieren und Einschätzen von Gestaltungsmerkmalen und Prozessen der Erzeugung medialer Botschaften, (3) Erkennen und Aufarbeiten von Medieneinflüssen auf Individuum und Gesellschaft, (4) Durchschauen und Beurteilen von Bedingungen der Medienproduktion und Medienverbreitung (Tulodziecki, Herzig, und Grafe 2019, 207-13).

Das skizzierte Konzept von Medienbildung führt - wie oben angesprochen - zu bestimmten Anforderungen an die Kompetenz und Professionalisierung von Lehrpersonen.

\section{Kompetenzerwartungen an Lehrpersonen auf der Grundlage des Diskurses um medienpädagogische Kompetenz}

Die oben genannten Aufgabenfelder der Medienbildung verweisen zunächst auf verschiedene Kompetenzerwartungen an die Schülerinnen und Schüler. Damit Lehrpersonen in der Lage sind, die Lernenden entsprechend zu fördern, müssen sie ihr Wissen und Können sowie ihre Einstellungen zur Umsetzung der Medienbildung in einem Prozess der Professionalisierung kontinuierlich reflektieren und aktualisieren. Dies setzt eine Klärung der Kompetenzerwartungen an Lehrpersonen voraus.

Zunächst ist für Lehrpersonen eine hinreichende allgemeine Medienkompetenz bezüglich der obigen nutzungs- und inhaltsbezogenen Aufgabenfelder notwendig - wobei zu bedenken ist, dass für viele Lehrpersonen bisher nur begrenzte Möglichkeiten bestanden und bestehen, die wünschenswerte Medienkompetenz als erste Voraussetzung für die Umsetzung der Medienbildung zu erwerben. Ausserdem sind aufgrund der rasanten Entwicklung der Informations- und Kommunikationstechnologien immer wieder Aktualisierungen einzufordern. Darüber hinaus verlangt die Umsetzung der Medienbildung Bereitschaften sowie Wissen und Können 
in verschiedenen Kompetenzbereichen, die für die Planung, Durchführung und Evaluation von medienpädagogischen Aktivitäten in der Schule notwendig sind. Entsprechende Überlegungen kommen im deutschsprachigen Bereich vor allem bei Ansätzen zur so genannten (medienpädagogischen Kompetenz〉 zur Geltung (Blömeke 2000; Gysbers 2008; Tulodziecki 2012; Herzig und Martin 2018).

Es ist hier nicht der Raum, eine Diskussion der Vorzüge und Probleme der verschiedenen Ansätze zu leisten. Deshalb stellen wir vor dem Hintergrund vorhandener Ansätze nur in aller Kürze wichtige Aufgabenfelder und Kompetenzerwartungen für die Lehrerinnen- und Lehrerbildung zusammen, wobei uns u.a. der - auch von Dorothee M. Meister miterarbeitete - Kompetenzorientierte Rahmen zur Entwicklung von Curricula für medienpädagogische Studiengänge und Studienanteile als Hintergrundfolie dient (DGfE-Sektion Medienpädagogik 2017). Auf dieser Grundlage können als wichtige Aufgabenfelder für die Lehrerinnen und Lehrerbildung - basierend auf einem entsprechenden Reflexions- und Gestaltungswissen gelten:

- Weiterentwicklung der eigenen Medienkompetenz im Sinne der Aktualisierung und (gegebenenfalls) der Aufarbeitung von Defiziten,

- Nutzung von Medien bzw. digitalen Umgebungen für Lernprozesse,

- Wahrnehmung von medienbezogenen Erziehungs- und Bildungsaufgaben,

- Entwicklung schulspezifischer Konzepte zur Medienbildung. 
Bezogen auf diese Aufgabenfelder orientieren wir uns bei der Formulierung von Kompetenzerwartungen an Aspekten, die für ein fundiertes Handeln generell bedeutsam sind (Tulodziecki 2017, 63):

- Analyse von Bedingungen des Handelns und ihre Beachtung,

- Analyse und Bewertung von Konzepten, Theorien und Forschungsergebnissen als Bezugspunkte für Handeln,

- Analyse und Bewertung vorhandener Beispiele des Handelns,

- Entwicklung eigener konzeptioneller Vorstellungen und Entwürfe zur Umsetzung,

- Erprobung und Evaluation eigener Entwürfe.

Entsprechende Kompetenzerwartungen sind in der Tabelle 1 für die genannten Aufgabenfelder aufgeführt.

\begin{tabular}{|l|l|}
\hline Aufgabenfelder & Kompetenzerwartungen \\
\hline $\begin{array}{l}\text { Weiterentwicklung } \\
\text { enkompenen Medi- }\end{array}$ & $\begin{array}{l}\text { Defizite bei der eigenen Medienkompetenz erkennen und auf- } \\
\text { arbeiten }\end{array}$ \\
$\begin{array}{l}\text { Entwicklungen im Medienbereich verfolgen und sich in neue } \\
\text { Entwicklungen, die für Lernen, Erziehung oder Bildung relevant } \\
\text { en bzw. digitalen } \\
\text { Lernumgebungen } \\
\text { für Lernprozesse }\end{array}$ & $\begin{array}{l}\text { sind, einarbeiten } \\
\text { tern }\end{array}$ \\
& $\begin{array}{l}\text { Aür das Lernen mit Medien relevant sind, beschreiben und im } \\
\text { Unterricht beachten } \\
\text { Konzepte, Theorien und empirische Forschungsergebnisse zum } \\
\text { Lehren und Lernen mit Medien bzw. in digitalen Umgebungen } \\
\text { skizzieren und in ihrer Bedeutung für die eigene Praxis ein- } \\
\text { schätzen } \\
\text { Medienangebote bzw. digitale Umgebungen für das Lernen } \\
\text { analysieren und bewerten } \\
\text { einfache Medienbeiträge zur Anregung, Unterstützung oder } \\
\text { Kontrolle von Lernprozessen selbst entwerfen und gestalten } \\
\text { vorhandene Beispiele zur Medienverwendung für Lernen analy- } \\
\text { sieren und bewerten } \\
\text { eigene Unterrichtseinheiten oder Projekte mit der Nutzung von } \\
\text { Medien bzw. von digitalen Lernumgebungen entwerfen, erpro- } \\
\text { ben und evaluieren }\end{array}$ \\
\hline
\end{tabular}




\begin{tabular}{|l|l|}
\hline $\begin{array}{l}\text { Wahrnehmung } \\
\text { von medienbezo- } \\
\text { genen Erziehungs- } \\
\text { und Bildungsauf- } \\
\text { gaben }\end{array}$ & $\begin{array}{l}\text { Grundbegriffe und Fragestellungen zu Erziehungs- und Bil- } \\
\text { dungsaufgaben im Medienbereich erläutern } \\
\text { Aspekte der Mediensozialisation von Kindern und Jugendli- } \\
\text { chen, die für Erziehung und Bildung relevant sind, beschreiben } \\
\text { und im Unterricht beachten } \\
\text { Ergebnisse und Methoden der Medienforschung, die sich als } \\
\text { Themen für die Medienbildung eignen, skizzieren und bewerten } \\
\text { Konzepte, Theorien und empirische Forschungsergebnisse zu } \\
\text { medienbezogenen Erziehungs- und Bildungsaufgaben erläutern } \\
\text { und in ihrer Bedeutung für die eigene Praxis einschätzen } \\
\text { Medienangebote, die für Kinder und Jugendliche interessant } \\
\text { sind, analysieren und bewerten } \\
\text { vorhandene Unterrichtseinheiten oder Projekte zu medienbe- } \\
\text { zogenen Erziehungs- und Bildungsaufgaben analysieren und } \\
\text { bewerten } \\
\text { eigene Unterrichtseinheiten oder Projekte zu medienbezoge- } \\
\text { nen Erziehungs- und Bildungsaufgaben entwerfen, erproben } \\
\text { und evaluieren }\end{array}$ \\
\hline $\begin{array}{l}\text { Entwicklung } \\
\text { schulspezifischer } \\
\text { Konzepte zur Me- } \\
\text { dienbildung }\end{array}$ & $\begin{array}{l}\text { istitionelle Bedingungen (personale, curriculare, organisato- } \\
\text { Medien skizzieren } \\
\text { vorhandene Konzepte zur medienbezogenen Organisation und } \\
\text { Entwicklung in der Schule skizzieren und in ihrer Bedeutung für } \\
\text { eigenes schulisches Handeln einschätzen } \\
\text { Beispiele für die Entwicklung schulspezifischer Konzepte im } \\
\text { Rahmen von Schulentwicklungsprozessen analysieren und } \\
\text { bewerten } \\
\text { ein schulspezifisches Konzept zur Medienbildung zusammen } \\
\text { mit anderen entwerfen und - wenn möglich - umsetzen und } \\
\text { evaluieren }\end{array}$ \\
\hline
\end{tabular}

Tab. 1.: Aufgabenfelder und Kompetenzerwartungen für die Lehrerinnen- und Lehrerbildung zur Umsetzung von Medienbildung (nach Tulodziecki, Herzig, und Grafe 2019, 363).

Neben deutschsprachigen Ansätzen zu〈medienpädagogischer Kompetenz〉 gibt es im internationalen Raum unterschiedliche Ansätze zur Beschreibung von Kompetenzen für Lehr- und Erziehungspersonen angesichts der informations- und kommunikationstechnologischen Entwicklungen. Ein Blick darauf kann helfen, die obigen Kompetenzerwartungen im Vergleich einzuschätzen und gegebenenfalls auf Anregungen aufmerksam zu werden. 


\section{Medienbezogene Kompetenzvorstellungen zur Lehrerinnen- und Lehrerbildung im internationalen Raum}

Ein international häufig zitierter Ansatz zu der Frage, über welches Wissen (knowledge) Lehrpersonen für eine reflektierte Verwendung technischer Medien (thoughtful pedagogical uses of technologY) verfügen müssen, ist das Konzept einer Verbindung von technischem bzw. technologischem, didaktischem bzw. pädagogischem und fachinhaltlichem Wissen (technological pedagogical content knowledge, TPCK) (Mishra und Koehler 2006, 1017). Dabei werden zunächst drei Wissensarten unterschieden: Wissen zu den Fakten, Konzepten, Theorien und Vorgehensweisen der jeweils zu unterrichtenden Disziplin; Wissen zu Prozessen und Methoden des Lehrens und Lernens unter Beachtung von Voraussetzungen und Bedingungen sowie Zielen und übergreifenden pädagogischen Leitideen; Wissen bezüglich der medialen Möglichkeiten - vom Buch bis zu digitalen Medien - und ihrer sachgerechten Nutzung im Unterricht. Diese drei Wissensarten werden im TPCK zusammengeführt, wobei eine Synthese entstehen soll, die über die einzelnen Wissensarten hinausgeht. Insgesamt geht es dabei um ein Verständnis des komplexen Zusammenhangs von Merkmalen der Lernenden, Zielen, Inhalten, Methoden, Medien und Eigenschaften von Lehrpersonen sowie um die situationsbezogene Berücksichtigung des Zusammenhangs bei der Vorbereitung, Durchführung und Evaluation von Unterricht (vgl. ebd., 1028-1029). Der TCPK-Ansatz erinnert u.a. an frühere deutschsprachige Ansätze zur Didaktik und Mediendidaktik mit ihrem Aufweis eines interdependenten Zusammenhangs von Intentionalität, Thematik, Methodik und Medienwahl im Verhältnis zu anthropogenen und sozial-kulturellen Voraussetzungen (Heimann, Otto, und Schulz 1965; Hagemann und Tulodziecki 1978).

Weitere internationale Überlegungen spiegeln sich u.a. im Media and Information Literacy Curriculum (MIL) Framework for Teachers der UNESCO wider (Wilson u. a. 2011). Darin werden drei miteinander in Beziehung stehende thematische Bereiche differenziert: Wissen und Verstehen von Medien und Information in ihrer Bedeutung für demokratische Diskurse und soziale Partizipation; Bewerten von Medientexten und Informationsquellen; Produktion und Nutzung von Medien und Information. Diese grundlegenden Bereiche werden mit verschiedenen generellen Aspekten von 
Erziehung und Lehrerinnen- und Lehrerbildung in ein weiterführendes Verhältnis gebracht (ebd., 23-29). Insgesamt ist der UNESCO-Ordnungsrahmen durch eine Akzentsetzung bei der Nutzung von Medien und Informationstechnologien für Lernen und Lehren im Sinne einer Förderung von Demokratie und Partizipation gekennzeichnet, gepaart mit der Erwartung, dass die medialen Möglichkeiten auch für die eigene Professionalisierung genutzt werden.

Einen weiteren Beitrag zu Kompetenzerwartungen an pädagogische Berufe stellt - mit besonderem Blick auf die Digitalisierung - der Europäische Kompetenzrahmen für die Digitale Kompetenz Lehrender (DigCompEdu) der Europäischen Kommission dar (Redeker und Punie 2017). Er ist für Personen aller Erziehungs- und Bildungsstufen sowie -formen gedacht und bezieht sich auf sechs Kompetenzbereiche: berufliches Engagement, digitale Ressourcen, Lehren und Lernen, Leistungsbeurteilung, Stärkung der Lernenden sowie Förderung der digitalen Kompetenz von Lernenden, wobei den Bereichen jeweils elementare Kompetenzen zugeordnet sind (ebd., 16-17). Auch dieser Kompetenzrahmen ist in besonderer Weise auf das Lehren und Lernen mit Medien konzentriert. Dabei werden Erziehende und Lehrende vor allem in ihrer Förderungsfunktion für das Lernen mit einer effektiven Nutzung von digitalen Technologien gesehen.

Insgesamt unterscheiden sich sowohl das MIL-Curriculum Framework for Teachers als auch der europäische DigCompEdu-Kompetenzrahmen von der deutschsprachigen Diskussion um medienpädagogische Kompetenz durch (a) ihre stärkere Fokussierung auf das Lehren und Lernen mit (digitalen) Medien, (b) ihre Vermischung der notwendigen (allgemeinen) Medienkompetenz von Lehr- und Erziehungspersonen mit der darüber hinaus gehenden pädagogischen Kompetenz und (c) ihre geringere Betonung von medienbezogenen Erziehungs-, Bildungs- und Schulentwicklungsaufgaben. Gleichzeitig integrieren sie deutlicher Fragen der Nutzung von digitalen Medien für die eigene Professionalisierung. Dieser kurze Vergleich verweist aus unserer Sicht zum einen auf gewisse Vorzüge der deutschsprachigen Diskussion. Zum anderen macht er aber (noch einmal) darauf aufmerksam, wie wichtig es ist, die Frage medienpädagogischer Kompetenz in den Kontext von Professionalisierungsüberlegungen zu stellen. 


\section{Kompetenzerwerb und Professionalisierung}

In der Ausbildungs- und Fortbildungspraxis von Lehrpersonen wird bezüglich des Kompetenzerwerbs zum Teil unterstellt, dass es hinreichend sei, medienpädagogisch relevante Kenntnisse, Fertigkeiten sowie Fähigkeiten zu (vermitteln〉, und dass dies dazu führe, dass das 〈Vermittelte〉 anschliessend in der Unterrichtspraxis mehr oder weniger problemlos angewendet würde. Solche Annahmen greifen allerdings zu kurz. Vielmehr setzt die Übertragung von Gelerntem auf neue Situationen ein kontextbezogenes und situiertes Lernen voraus. Dies verweist erneut darauf, wie wichtig es ist, den Erwerb medienpädagogischer Kompetenz im Kontext von Überlegungen zur Professionalisierung zu betrachten (Knaus, Meister, und Tulodziecki 2018, 30-34). Eine entsprechende Rahmung bedeutet u.a., dass der Kompetenzerwerb an Merkmalen professionellen Handelns orientiert sein sollte und dass die Entwicklung zu einem wünschenswerten beruflichen Handeln unter einer längerfristigen Perspektive zu sehen ist.

In diesem Zusammenhang lässt sich professionelles Handeln als Bereitschaft und Fähigkeit zu einem situationsangemessenen, wissenschaftlich fundierten und verantwortungsbewussten beruflichen Handeln beschreiben. Ein solches Handeln setzt voraus: (a) die Verfügung über wissenschaftliche Grundlagen für das Handeln, (b) die Fähigkeit, wissenschaftliche Grundlagen fallbezogen mit praktischen Situationen in Beziehung zu setzen, (c) eine forschende Grundhaltung, (d) weitere Persönlichkeitseigenschaften, die für die Wahrnehmung beruflicher Aufgaben förderlich sind. Dabei sollten wissenschaftliche Grundlagen sowohl theoretische Ansätze als auch empirische Ergebnisse und forschungsmethodische Vorgehensweisen umfassen. Ein fallbezogenes In-Beziehung-Setzen von wissenschaftlichem Wissen mit praktischen Situationen ist aufgrund der besonderen Strukturen pädagogischen Handelns notwendig. Strukturell geht es bei professionellem Handeln um ein Spannungsverhältnis zwischen situativen Gegebenheiten und allgemein formuliertem Regelwissen. Beides muss bei konkreten pädagogischen Anforderungen auf der Grundlage von Fallverstehen miteinander in Verbindung gebracht und zu pädagogisch verantwortlichen Entscheidungen zusammengeführt werden. Dies sollte sowohl in der Vorbereitung pädagogischer Aktivitäten als auch während der Aktivitäten und im Anschluss an diese geschehen. Eine forschende 
Grundhaltung ist dadurch gekennzeichnet, dass sowohl die Bedingungen des Handelns als auch das eigene Handeln selbst kritisch begleitet und evaluiert werden. Wichtige Persönlichkeitseigenschaften lassen sich unter dem Begriff einer berufsethischen Haltung zusammenfassen, die bei Lehrpersonen vor allem durch Respekt und Verantwortungsbewusstsein für Kinder und Jugendliche geprägt sein sollte. Bezüglich wichtiger Haltungen geht es im Medienbereich zudem um eine Reflexion der eigenen Einstellung gegenüber Medien (Kommer und Biermann 2012).

Gemäss diesen Merkmalen professionellen Handelns ist es für den Erwerb medienpädagogischer Kompetenz wichtig, eine reflexive Auseinandersetzung mit wissenschaftlichen Grundlagen zu leisten, diese immer wieder in Beziehung zu praktischen Situationen zu setzen, eine forschende Grundhaltung aufzubauen sowie berufsethische Fragen und eigene Einstellungen zu bedenken. Im Kontext solcher Prozesse geht es auch darum, das Verhältnis von Theorie und Praxis zu reflektieren. Dabei sollten beide Bereiche nicht - wie es manchmal geschieht - als getrennte Domänen aufgefasst, sondern durch das angesprochene In-Beziehung-Setzen von wissenschaftlichen Grundlagen mit praktischen Fällen bzw. Situationen in Verbindung gebracht werden (Tulodziecki, Herzig, und Blömeke 2017, 322).

Darüber hinaus gilt - wie oben angesprochen -, dass professionelles Handeln nicht in zeitlich eng begrenzten Phasen der Auseinandersetzung mit medienpädagogisch relevanten Themen erreicht werden kann, sondern ein Denken in grösseren Zeiträumen erfordert. Darauf verweisen insbesondere Ansätze zur Expertiseforschung (Berliner 1994; Neuweg 1999). Danach muss Professionalisierung als Entwicklungsvorgang aufgefasst werden, der vom Anfängerstadium (Novice) über die Zwischenstufe eines fortgeschrittenen Anfängertums (Advanced Beginner) und die Stufe des kompetenten Handelns (Competent Level of Performance) sowie über eine weitere Zwischenstufe (Proficient Level) zum Expertenstatus (Expert) führt. Auf der Novizenstufe besteht das Problem darin, dass die Handelnden zwar über (kontextfreies) Wissen verfügen, während des pädagogischen Handelns jedoch kaum freie kognitive Kapazitäten haben, um in Handlungssituationen Analysen vorzunehmen und darauf basierende Entscheidungen zu treffen. Dies gelingt erst mit zunehmender Erfahrung, auf deren Basis sich später die Stufe des kompetenten Handelns herausbilden kann. Auf 
dieser Stufe können in Reaktion auf aktuelle Ereignisse angemessene Entscheidungen getroffen werden - gegebenenfalls auch abweichend von der Planung. Zugleich haben sich erste situationsangemessene Routinen herausgebildet. Mit der Weiterentwicklung entsprechender Fähigkeiten kann sich schliesslich eine immer stärkere situationsspezifische Organisation des Wissens einstellen, verbunden mit einem zunehmend schnellen und routinierten Zugriff darauf. Dies führt auf der Expertenstufe sowohl vor als auch während einer Handlung und in nachfolgenden Reflexionen stets zu situations- und fallangemessenen sowie verantwortungsbewussten Analysen, Bewertungen und Entscheidungen.

Auf der Grundlage einer solchen Modellvorstellung sind erste Unterrichtsversuche von Studierenden dem Novizenstadium zuzuordnen. Bei günstigen Bedingungen, z.B. bei hinreichenden unterrichtspraktischen Erfahrungen mit erfahrungs- und handlungsorientierten Lernformen, kann sich zum Abschluss des Studiums ein fortgeschrittenes Anfängerstadium und zum Ende von Referendariat und einer anschliessenden Berufseingangsphase die Stufe kompetenten Handelns ausbilden. Dabei ist der Kompetenzerwerb allerdings - bezogen auf das der Entwicklungsvorstellung zugrunde liegende Kompetenzverständnis - noch nicht hinreichend für das letztlich angestrebte professionelle Handeln. Dieses bedarf längerfristiger Berufserfahrungen mit einer begleitenden reflexiven Verarbeitung der jeweiligen Erfahrungen unter Berücksichtigung wissenschaftlicher Erkenntnisse. So lässt sich schliesslich die Expertenstufe erreichen.

\section{Literatur}

Berliner, David C. 1994. «Teacher expertise». In The international encyclopedia of education, herausgegeben von Torsten Husen und Thomas N. Postlethwaite, 2. Edition, 10:6020-26. London: Pergamon.

Blömeke, Sigrid. 2000. Medienpädagogische Kompetenz. Theoretische und empirische Fundierung eines zentralen Elements der Lehrerausbildung. München: kopaed.

DGfE-Sektion Medienpädagogik. 2017. «Orientierungsrahmen für die Entwicklung von Curricula für medienpädagogische Studiengänge und Studienanteile». MedienPädagogik:Zeitschriftfür Theorie Und Praxis Der Medienbildung (Statements and Frameworks), 1-7. https://doi.org/10.21240/mpaed/oo/2017.12.04.X.

Döbeli Honneger, Beat. 2016. Mehr als O und 1. Schule in einer digitalisierten Welt. Bern: hep. 
Gapski, Harald. 2016. «Medienkompetenz 4.0? Entgrenzungen, Verschiebungen und Überforderungen eines Schlüsselbegriffs». merz medien + erziehung. Zeitschrift für Medienpädagogik 60 (4): 19-25.

Gysbers, André. 2008. Lehrer, Medien, Kompetenz. Eine empirische Untersuchung zur medienpädagogischen Kompetenz und Performanz niedersächsischer Lehrkräfte. Berlin: VISTAS.

Hagemann, Wilhelm, und Gerhard Tulodziecki. 1978. Einführung in die Mediendidaktik. Köln: Verlag Schulfernsehen.

Heimann, Paul, Gunter Otto, und Wolfgang Schulz. 1965. Unterricht. Analyse und Planung. Hannover: Schrödel.

Herzig, Bardo. 2012. Medienbildung. Grundlagen und Anwendungen. Bd. 1. Handbuch Medienpädagogik. München: kopaed.

Herzig, Bardo, und Alexander Martin. 2018. «Lehrerbildung in der digitalen Welt». In Digitalisierung und Bildung, herausgegeben von Silke Ladel, Julia Knopf, und Armin Weinberger, 89-113. Wiesbaden: Springer Fachmedien Wiesbaden. https://doi.org/10.1007/978-3-658-18333-2_6.

Knaus, Thomas. 2016. «digital - medial - egal?» In Kommunikationskulturen in digitalen Welten: Konzepte und Strategien der Medienpädagogik und Medienbildung, herausgegeben von Marion Brüggemann, Thomas Knaus, und Dorothee M. Meister, 99-130. Schriften zur Medienpädagogik 52. München: kopaed.

Knaus, Thomas, Dorothee M. Meister, und Gerhard Tulodziecki. 2018. «Qualitätsentwicklung - Professionalisierung - Standards. Thesen aus medienpädagogischer Sicht». In Futurelab Medienpädagogik. Qualitätsentwicklung. Professionalisierung. Standards, 23-47. Schriften zur Medienpädagogik 45. München: kopaed.

Kommer, Sven, und Ralf Biermann. 2012. «Der mediale Habitus von (angehenden) LehrerInnen. Medienbezogene Dispositionen und Medienhandeln von Lehramtsstudierenden». In Jahrbuch Medienpädagogik 9, herausgegeben von Renate Schulz-Zander, Birgit Eickelmann, Heinz Moser, Horst Niesyto, und Petra Grell, 81-108. Wiesbaden: VS Verlag für Sozialwissenschaften. https:// doi.org/10.1007/978-3-531-94219-3_5.

Krotz, Friedrich. 2016. «Wandel von sozialen Beziehungen, Kommunikationskultur und Medienpädagogik. Thesen aus der Perspektive des Mediatisierungsansatzes». In Kommunikationskulturen in digitalen Welten, herausgegeben von Marion Brüggemann, Thomas Knaus, und Dorothee M. Meister, 19-42. München: kopaed.

Krotz, Friedrich, und Andreas Hepp. 2012. «Mediatisierte Welten. Forschungsfelder und Beschreibungsansätze - Zur Einleitung». In Mediatisierte Welten. Forschungsfelder und Beschreibungsansätze, herausgegeben von Friedrich Krotz und Andreas Hepp, 7-23. Wiebaden: VS.

Meister, Dorothee M., und Uwe Sander. 1999. «Multimedia und Kompetenz». In Multimedia: Chancen für die Schule, herausgegeben von Dorothee M. Meister und Uwe Sander, 35-53. Praxishilfen Schule Pädagogik. Neuwied: Luchterhand. 
Mishra, Punya, und Matthew J. Koehler. 2006. «Technological Pedagogical Content Knowledge: A Framework for Teacher Knowledge». Teachers College Record 108 (6): 1017-54. https://doi.org/10.1111/j.1467-9620.2006.00684.x.

Neuweg, Georg Hans. 1999. «Erfahrungen in der LehrerInnenbildung - Potenziale und Grenzen im Lichte des Dreyfus-Modells». Erziehung und Unterricht 5/6: 363-72.

Redeker, Christine, und Yves Punie. 2017. «European Framework for the Digital Competence of Educators: DigCompEdu». Luxembourg: Publications Office of the European Union.

Tulodziecki, Gerhard. 2012. «Medienpädagogische Kompetenz und Standards in der Lehrerbildung». In Jahrbuch Medienpädagogik 9, herausgegeben von Renate Schulz-Zander, Birgit Eickelmann, Heinz Moser, Horst Niesyto, und Petra Grell, 271-97. Wiesbaden: VS Verlag für Sozialwissenschaften. https://doi. org/10.1007/978-3-531-94219-3_13.

Tulodziecki, Gerhard. 2017. «Thesen zu einem Rahmenplan für ein Studium der Medienpädagogik». merz medien + erziehung. Zeitschrift für Medienpädagogik 61 (3): 59-65.

Tulodziecki, Gerhard, Bardo Herzig, und Silke Grafe. 2019. Medienbildung in Schule und Unterricht: Grundlagen und Beispiele. 2. Aufl. Bad Heilbrunn: Klinkhardt/ UTB.

Tulodziecki, Gerhard, Bardo Herzig, und Sigrid Blömeke. 2017. Gestaltung von Unterricht: eine Einführung in die Didaktik. 3., überarbeitete und erweiterte Auflage. UTB Erziehungswissenschaft, Schulpädagogik, Allgemeine Didaktik 3311. Bad Heilbrunn: Verlag Julius Klinkhardt.

Wilson, Carolyn, Alton Grizzle, Ramon Tuazon, Kwame Akyempong, und ChiKim Cheung. 2011. «Media and Information Curriculum for Teachers». Paris: UNESCO. 